\title{
Artificial Neural Network Approach to Mobile Location Estimation in GSM Network
}

\author{
Longinus S. Ezema and Cosmas I. Ani
}

\begin{abstract}
The increase in utilisation of mobile location-based services for commercial, safety and security purposes among others are the key drivers for improving location estimation accuracy to better serve those purposes. This paper proposes the application of Levenberg Marquardt training algorithm on new robust multilayered perceptron neural network architecture for mobile positioning fitting for the urban area in the considered GSM network using received signal strength (RSS). The key performance metrics such as accuracy, cost, reliability and coverage are the major points considered in this paper. The technique was evaluated using real data from field measurement and the results obtained proved the proposed model provides a practical positioning that meet Federal Communication Commission (FCC) accuracy requirement.
\end{abstract}

Keywords - Neural Network, Artificial Intelligent, Levenberg Marquardt, Multilayered Perceptron, Mobile Location Estimation, GSM

\section{INTRODUCTION}

$\mathbf{M}$ OBILE estimation location has recently been attracting interest as it offers us the opportunity to locate a mobile user while in emergency and it also allows the user utilise other location services available on wireless communication. In some countries, by law, every wireless cellular network operators are required to provide a level of accuracy for the location of every emergency caller. Most of the performance measure has been standardised by Federal Communication Commission (FCC) of United State of America (USA), a communication regulatory body in the US. Their regulations have come in phases with accuracy requirement of phase II currently in use while researchers are on their toes to meet the accuracy requirement of phase III realised February 2015 and expected to take effect in six years time [1,2].

These applications have varying accuracy requirement of the user's location, such application as E911 and E112 are emergency service with high demand for accuracy, unlike commercial applications.

There are many techniques applied by researcher utilising different or a combination of mobile cellular network parameters which include Received Signal Strength (RSS), Cell ID, Angle of Arrival (AOA) and time measurement such as Time Different of Arrival (TOA), Time Different of Arrival (TDOA), Enhanced Observed Time Difference (E-OTD), Time Advance/Round Tripp Time (TA/RTT) [4]. The flexibility and robustness of artificial neural network to adapt to different environments and its processing speed has been the reason for its adoption as an alternative technique in this paper.

L. S. Ezema is with Department of Electronics Engineering, University of Nigeria Nsukka, Enugu State, Nigeria (e-mail: ezemms@ yahoo.com).

C. I. Ani is with Department of Electronic Engineering, University of Nigeria Nsukka, Enugu State, Nigeria (e-mail: cosmas.ani@unn.edu.ng).

\section{ARTIFICIAL NeURAL NETWORK BASED LOCATION ESTIMATION APPROACH}

This section presents mobile location estimation approach based on neural network using real measured received signal strength in an urban environment. The unpredictable and unstable nature of propagation path loss (signal strength) especially in an urban area made mobile location prediction a difficult and a complex task. Many techniques mainly based on geometric and statistical methods have been proposed, but the results have not been satisfactory when compared with FCC standard and results obtained when other parameters are utilized.

\section{A. Architecture of a Neural Network}

A well trained artificial neural network can perform many complex tasks such as classification, optimization, control and approximation function. Neural network can also be used for pattern matching or to establish a relationship between two variables (example- the RSS sample and location coordinates). This pattern matching algorithm of neural network can also be viewed as a function approximation as used in this work An artificial Neural network (ANN) consist of processing units called neuron which communicate by sending signals to each other over a large number of weighted (w) connections, see figure 1 [5].

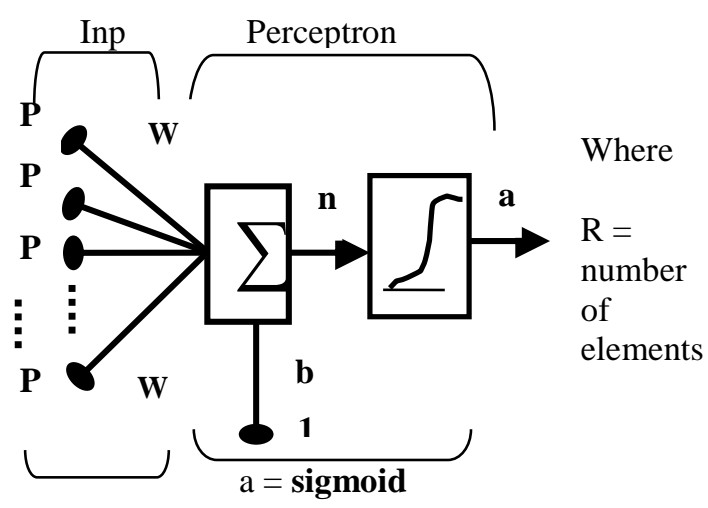

Fig. 1. Perceptron Neuron with sigmoid transfer function

Where, $\mathrm{p}$ is individual input, $\mathrm{b}$ is the neuron bias or offset, $\mathrm{w}$ is input weight, $\mathrm{n}$ is net input as the argument of the transfer function $f$, $a$ is output of a transfer function $(a=f(W p+b)$ ). The transfer function is sigmoid, see figure 2, takes input (n) ranging from minus infinity to positive infinity and output value in a range 0 to 1 using equation (1) below. 


$$
a=\frac{1}{1+e^{n}}
$$

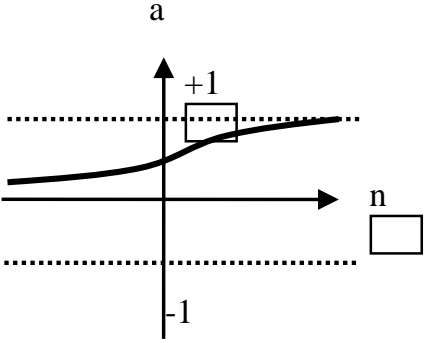

$a=\log \operatorname{sid}(n)$

\section{A. Multi-Layer Perceptron positioning algorithm}

Backpropagation is the generalization of the Widrow-Hoff learning rule to multiple layer perceptron (MLP) networks and nonlinear differentiable transfer functions [5]. The MLP is a feed-forward multi-layer network which uses a supervised error-based learning mechanism. This forward process is such that unites in one layer can only receive signal from unites directly below and sends output to unites directly above and no unites in the same layer are connected or can communicates. MLP is example of a static network without feedback or delays. The input vectors to the network are feed concurrently at same time or in no particular time sequence. In training the network, batch training with concurrent input vectors was adopted against incremental training. Batch training allows weights and biases to be updated only after all the inputs and targets have been presented. Although batch training can be trained with either 'adapt' or 'train', 'train' was used because it has access to more efficient training algorithms and generally the best option [5]. One pass of train with concurrent updating is called 'Epoch' or iteration. The data is divided into three, $70 \%$ is used for training while $15 \%$ each of the remaining $30 \%$ is used for validation and testing of the ANN model.

ANN are very sensitive to absolute magnitudes, the inputs ranges from $-48 \mathrm{~dB}$ to $-113 \mathrm{~dB}$ and others are very big values (the output-geographical coordinates), fluctuations in the big range input will tend to swamp any importance given to the first, even if the first input is much more important in predicting the desired output. In order to minimize the influence of absolute scale, all inputs to a network are scaled in our case and normalized so that they correspond to roughly the same range of values -1 to 1 . This pre-processing function transforms input data to a form that is easier or more efficient and that can speed up learning for neural networks. Similarly, the target outputs can also be pre-processed while the network outputs are reverse or post-processed using the same functions to produce output data with the same characteristics as the original user provided targets [5,6].

An Artificial Neural network (ANN) consist of processing units which communicate by sending signals to each other over a large number of weighted (w) connections. The total input to unit, $k$, is simply the weighted sum of the separate outputs from each of the connected units plus a bias or offset term $b_{k}$. in the MLP architecture shown in figure 3 below, the total input and the output of each neuron of the n-th layer is defined by equation (2) and equation (3) respectively $[9,10]$.

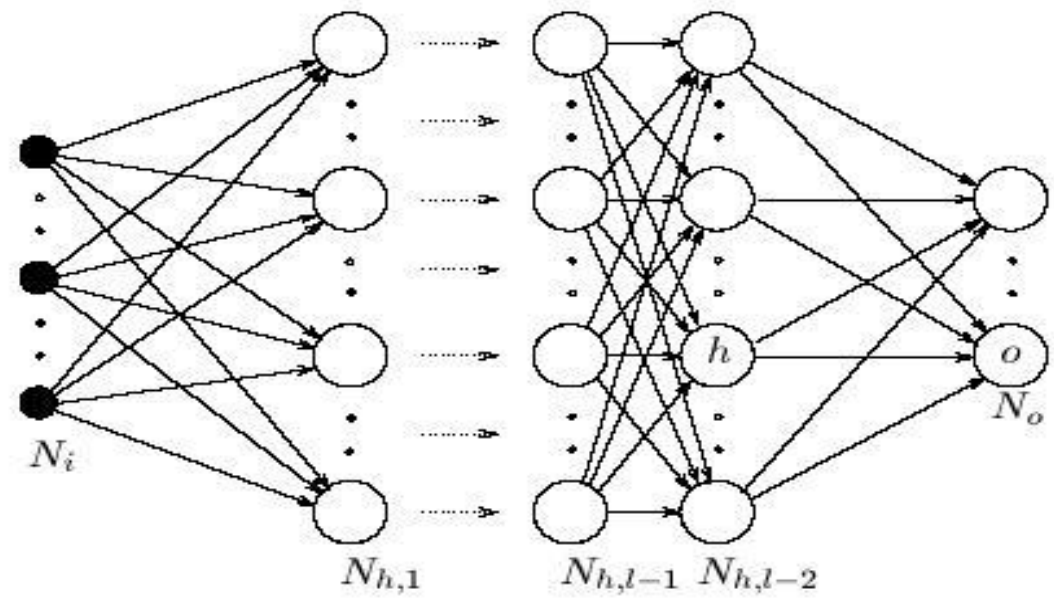

Fig. 3. MLP General Architecture

$$
\begin{gathered}
a_{k}=\sum_{j} w_{j k} y_{j}+b_{k} \\
y_{k}=F\left(\sum_{j} w_{j k} y_{j}+b_{k}\right)=F\left(a_{k}\right)
\end{gathered}
$$

Where $\mathrm{F}$ is the nonlinear activation function- sigmoid, $w_{j k}$ are the weights of the connection between the neuron $h_{j}$ and $h_{k}, y_{j}$ is the output of the neuron of the (n-th) layer. 
The algorithm used to adapt the weights in this work is Levenberg Marquardt for its simplicity and low memory requirement for generalization. The mean square error of the system is used as the performance index for multi-layer network training and is minimized by moving down to the gradient of the error curve. Levenberg-Marquardt algorithm is a mechanism used in optimising the network weights and biases by continuously modifying the weights in order to minimize the mean square error (MSE) between the target/desire output and the actual output of the network based on the equations below.

For every network input sample, the network output differs from the desired/target value $d^{p}$ by $\left(d^{p}-y^{p}\right)$ where $y^{p}$ is the actual network output for this input pattern. The delta-rule now uses an error-function based on these differences for weight adjustment. The error-function, as indicated by the name least mean square, is the summed squared error. That is, the total error $\mathrm{E}$ is defined as;

$$
\begin{aligned}
& E=\sum_{p} E^{p}=\frac{1}{2} \sum_{p}\left(d^{p}-y^{p}\right)^{2}, \\
& \Delta W=\left(J^{T} J+\mu I\right)^{-1} J^{T} e
\end{aligned}
$$

$$
W_{m+1}=W_{m}+\Delta W
$$

Where $\mathrm{e}$ is an error vector, $\Delta \mathrm{W}$ is change in weight, $\mu$ is a scalar parameter, $\mathrm{W}$ is a matrix of networks weights and $\mathrm{J}$ is the Jacobian matrix of the partial derivatives of the error components with respect to the weights. Also, $W_{n}$ is the old weight and $W_{n+1}$ is the new weight for the next iteration or the final weight of the network model. When the scalar $\mu$ is zero, this is just Newton's method, using approximate Hessian matrix in equation (7).

$$
\mathrm{H}=\mathbf{J}^{\mathrm{T}} \mathbf{J}
$$

It becomes gradient descent, equation (8) with a small step size when $\mu$ is large.

$$
\mathrm{g}=\mathrm{J}^{\mathrm{T}} \mathrm{e}
$$

Newton's method is faster and more accurate minimum error, so shifting to Newton's method as fast as possible will always be the aim of LMA $[5,9]$.

\section{PERFORMANCE EVALUATION OF LOCATION ESTIMATION TECHNIQUES}

In this section, the performance evaluation of the proposed artificial neural network model using real data collected from some of GSM BSs that cover the urban area of Achara layout in the city of Enugu, Nigeria is presented. Artificial Neural network as a pattern matching or approximation algorithm in this mobile location estimation is Multi-Layer perceptron (MLP) feed forward networks (see figure 5). It is one consisting of twelve inputs (RSS from twelve MS), one hidden layer of 22 neurons and an output layer with two neurons corresponding to location of MS $(\mathrm{x}, \mathrm{y})$. In order to run on the neural network architecture, Levenberg Marquardt algorithm written in Matlab code with the programme flow chat in figure 4 is applied. Three hundred and ninety six (396) samples measurements were taken and the data were divided into three in the ratio of 70:15:15 for training, validation and testing the developed position algorithm respectively. The training of the network is allowed to stop the moment the performance of validation set terminated or the performance goal is achieved. During the training, several network topologies were tried by changing the number of hidden layers and neurons in each layer. One hidden layer with 22 neurons, enough to produce minimal mean square error was found. The transfer functions for the neurons in the hidden and output layers are sigmoid and hard limit respectively.

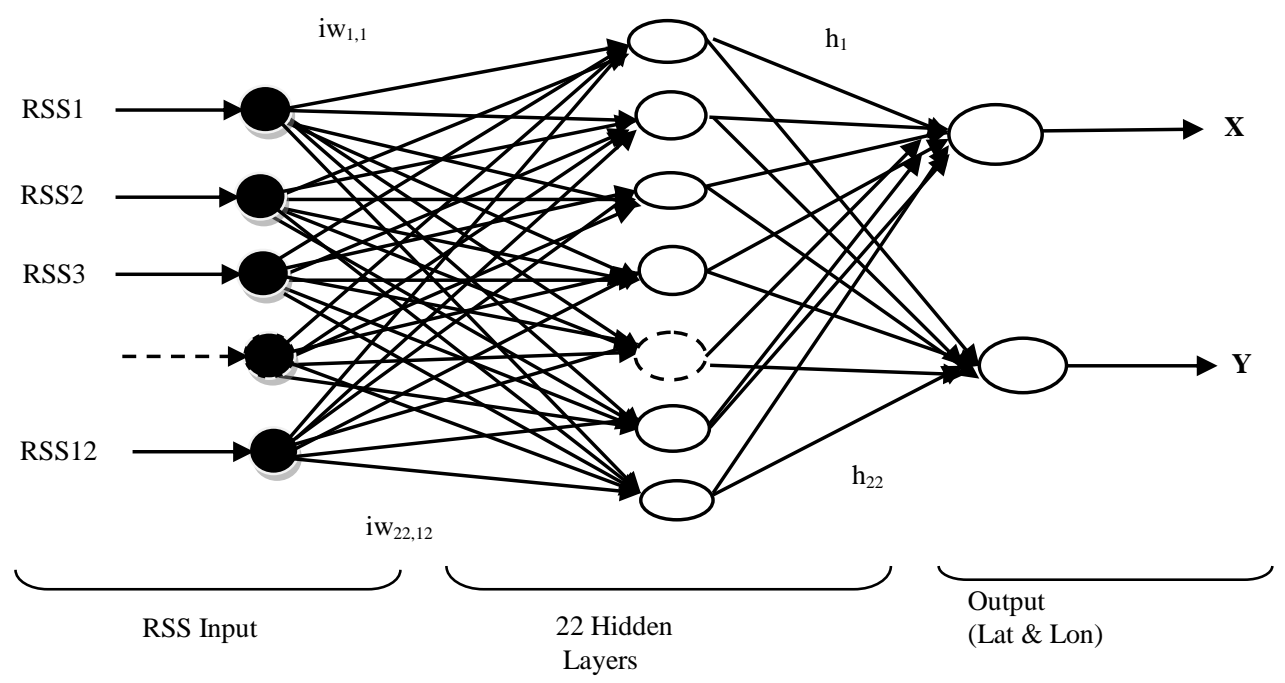

Fig. 5. Proposed Multi-Layered Perceptron architecture 


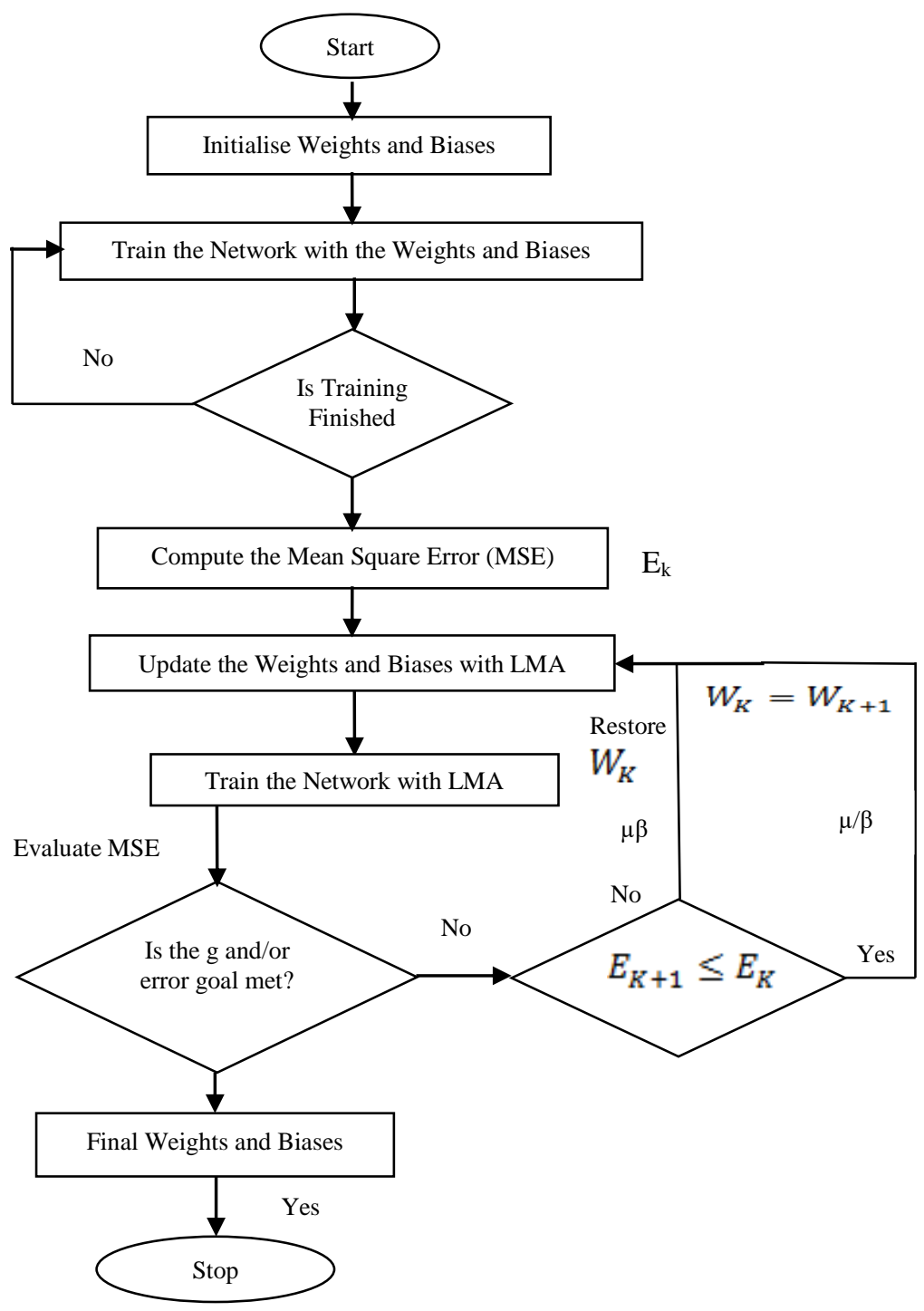

Fig. 4. LMA Flow Chart

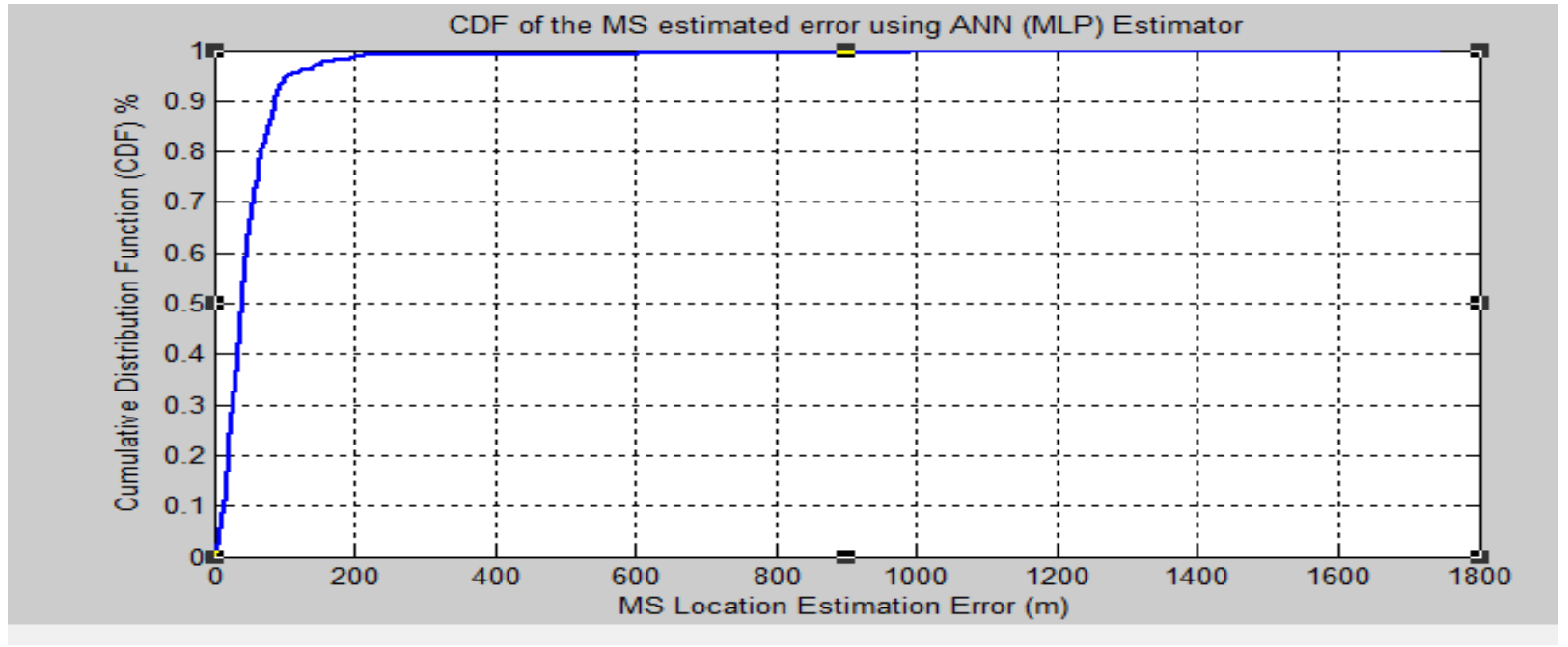

Fig. 6. CDFs of location estimation error for ANN (MLP)

For the network training and simulation MATLAB ${ }^{\circledR}$ neural network toolbox was used. 


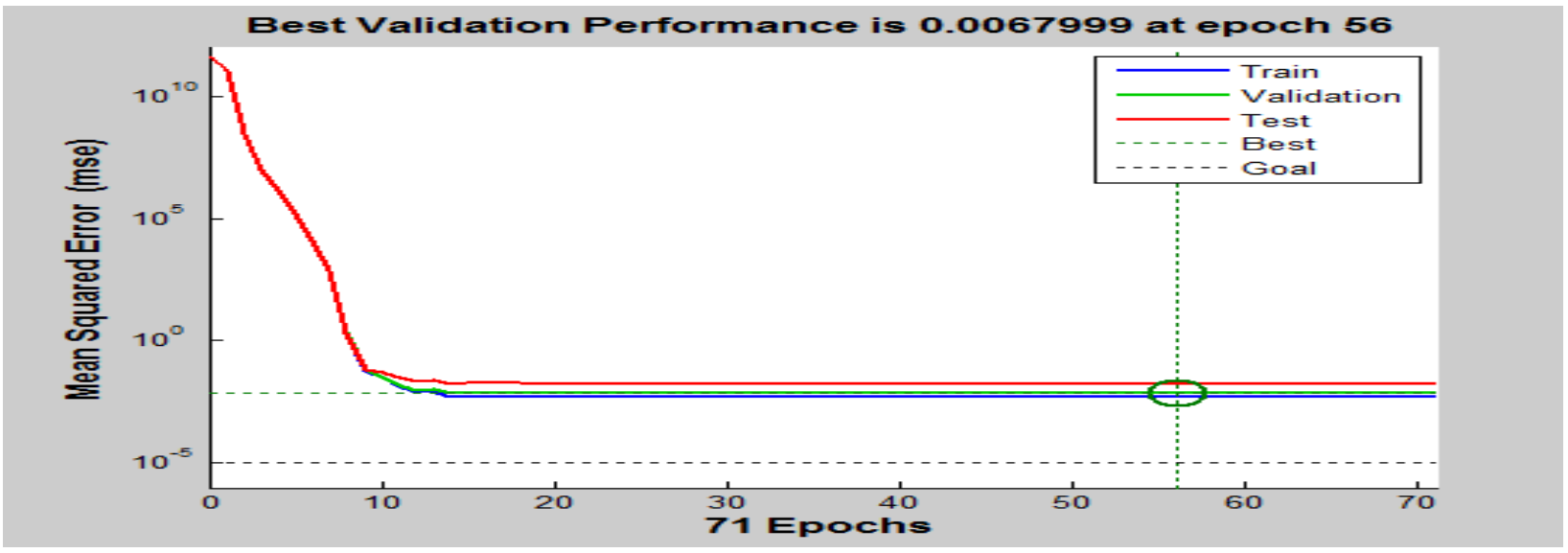

Fig. 7. Performance analysis of the network response

From figure 7 , the final mean-square error obtained during testing is $65 \mathrm{~m}$ and it was gotten at epoch 71 . The MSE was initially large at the start of the training session but reduces quickly as the training processes which show the learning progress of the network. Also the test set error and the validation set error has similar characteristics and no overfitting occurred at iteration 71 (where the best validation performance occurs). Training of the training vectors continues as long as MSE of the network reduces on the validation vectors. The training stops when the network memorizes the training set to avoid poor generalization. This technique ensures that the problem of over-fitting is avoided. The regression plots of the network training, validation and test are shown in figure 8 and figure 9.

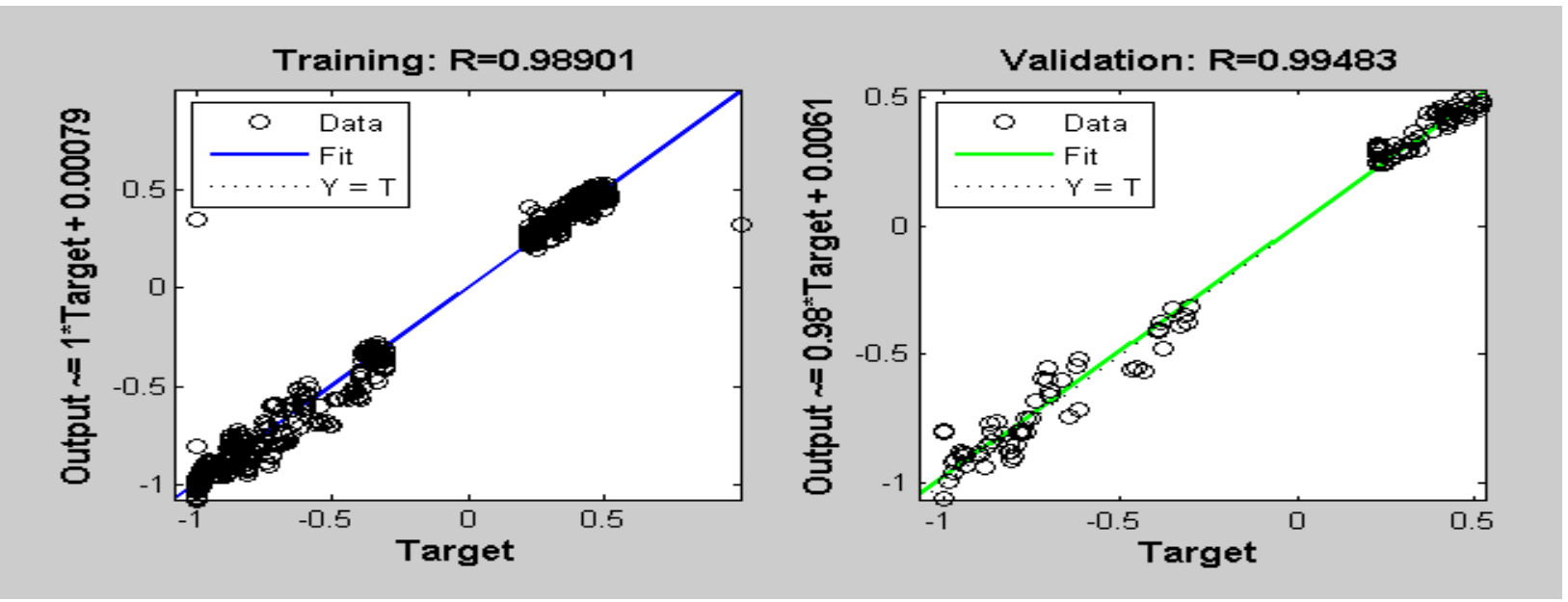

Fig. 8. Regression plot of output versus target for training data (left) and validation data (right).

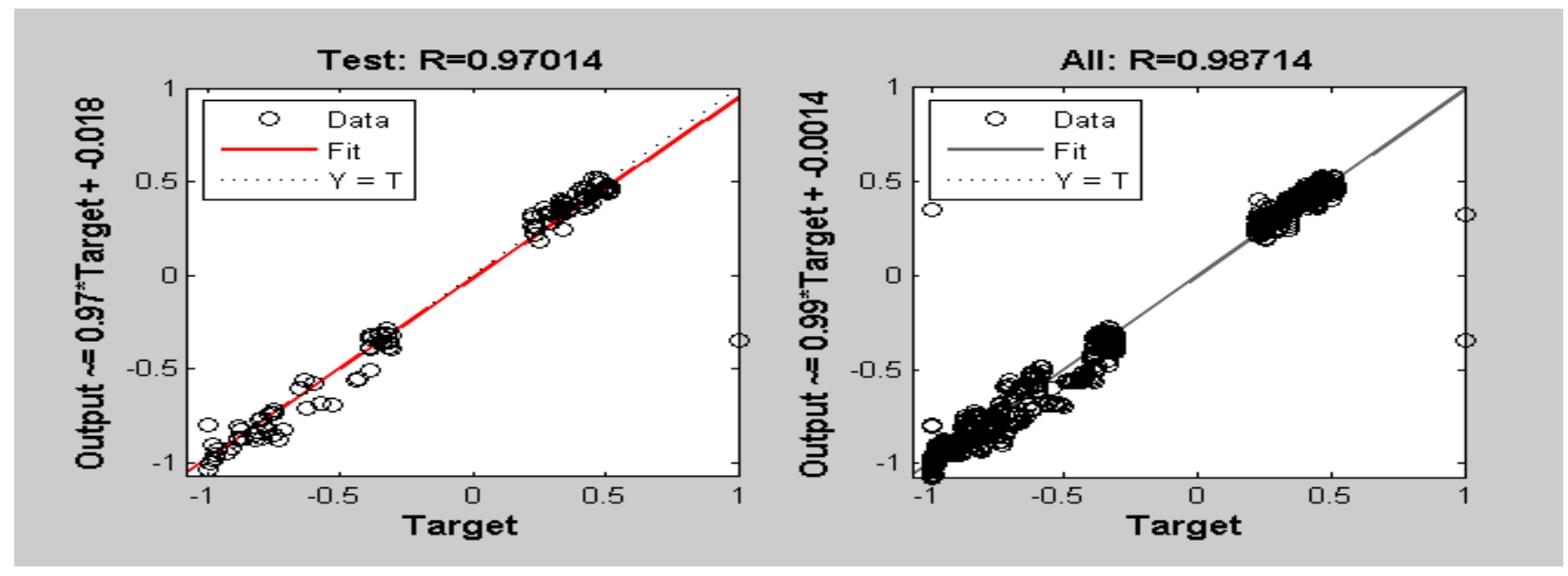

Fig. 9. Regression plot of output versus target for test data (left) and all data (right) for the generalised network. 
The regression plots show the correlation or linear regression between the network output and the corresponding targets values. An $\mathrm{R}$ value of 1 means a close relationship or output equals target while zero (1) means a random relationship. The closer the $\mathrm{R}$ value is to 1 the better the result. All the $\mathrm{R}$ values of the two figures 8 and figure 9 are above 0.97 (97\%). The reason the output tracks the targets very well for training, validation, and testing, and the R-value is over 0.98 for the total response.

The results of GSM-based mobile estimation accuracy are shown in table 1 (root mean square error (RMSE), mean, standard deviation (STD), $67 \%$ error and $95 \%$ error) in meters. The localisation error is calculated as the Euclidian distance between the actual position and the estimated position. Some of these performance metrics can easily be picked from the cumulative distribution function (CDF) graph in figure 6.

\section{Table I}

Summary Statistics of Simulation Result for from the Proposed Model

\begin{tabular}{|l|l|l|l|l|l|}
\hline Method & $\begin{array}{l}\text { RMSE } \\
(\mathrm{m})\end{array}$ & $\begin{array}{l}\text { STD } \\
(\mathrm{m})\end{array}$ & $\begin{array}{l}\text { MEAN } \\
(\mathrm{m})\end{array}$ & $67 \%(\mathrm{~m})$ & $95 \%(\mathrm{~m})$ \\
\hline MLP & 65 & 119 & 48 & 64 & 129 \\
\hline FCC & Nill & Nill & Nill & 100 & 300 \\
\hline
\end{tabular}

The table I above shows the simulation result obtained using real data from field measurement. Comparing the result with FFC accuracy requirement indicates how good the proposed method is and can be adopted for any location base services.

\section{CONCLUSION}

In this paper, the pattern learning approach to mobile location estimation by using neural network is presented. The performance evaluation results confirm the efficiency and the effectiveness of LMA for mobile positioning. This desired improvement represents a large increase in accuracy in comparison to FFC benchmark for emergency requirements. The mean square error of less than $64 \mathrm{~m}$, and $67 \%$ and $95 \%$ positioning error of less than $64 \mathrm{~m}$ and $129 \mathrm{~m}$ respectively show $36 \%$ and $57 \%$ improvement over the FCC minimum accuracy requirement respectively. One major advantage of this method is that there is no need for additional signaling, hardware or software installation in both mobile station and network. The highly availability of RSS in most cellular networks gives this method an edge over other LDP. Artificial Neural Network (ANN) model when applied with RSS reduces the uncertainty associated with RSS by learning from experience gained during training by using iterative algorithm.

\section{REFERENCE}

[1] M. B. Zeytinci, V. Sari, F. K. Harmanci, E. Anarim and M. Akar, "Location Estimation Using RSS Measurements with Unknown Path Loss Exponents", EURASIP Journal on Wireless Communications and Networking, 2013

[2] L.S. Ezema and C.I. Ani, "Multiple Linear Regression Model for Mobile Location Estimation in GSM Urban Environments", Indian Journal of Science and Technology; Feb., 2016

[3] M. Samiei, M. Mehrjoo and B. Pirzade, "Advances of Positioning Methods in cellular Networks", International Conference on Communications Engineering", December 2010

[4] L.S. Ezema, C.I. Ani and G.N. Ezeh, "Mobile Location Estimation in GSM/UMTS", International Journal of Emerging Technology \& Research, Vol. 1, Issue 3, Mar-Apr, 2014

[5] H. Demuth, M. Beale and M. Hagan, 'Neural Network Toolbox 6 User's Guide' The MathWorks, Inc, MA, US, March, 2009

[6] C. M. Takenga and K. Kyamakya, 'Location Fingerprinting in GSM Network and Impact of Data Preprocessing',

[7] M. Stella, M. Russo, and D. Begusic, 'GSM-Based Approach for Indoor Localisation', World Academy of Science, Engineering and Technology, 2013

[8] J. Muhammad, 'Artificial Neural Networks for location Estimation and Co-channel Interference Suppression in Cellular Networks', February, 2007

[9] S. Haykin, 'Neural Networks: A Comprehensive foundation', $2^{\text {nd }}$ Edition, Prentice Hall, 1998

[10] A. Hussain, 'Novel Artificial Neural Network Architecture and Algorithms for Non-Linear Dynamical Systems Modeling and Digital communications Applications', PhD Thesis, 1996

[11] J. Costabile, 'Wireless Position Location', Virginia tech Wireless symposium, June 4, 2010.

[12] J. Venkata Subramanian, and M. Abdul Karim Sadiq, "Implementation of Artificial Neural Network for Mobile Movement Prediction", Indian Journal of science and Technology; 7(6), pp. 858-863, June 2014

[13] Zoran Salcic, "AGPCS - An Automatic GSM-based Positioning and Communication System", Second annual conference of GeComputation, University of Otago, New Zealand, 26-29 August 1997 